\title{
Gendering Hypertensive Syndromes: Placentary Aspects and Umbilical Cords
}

Francisco Albuquerque Klank ${ }^{\star}$, José Ronaldo Alves Santos, Débora Lima, Jonas Pinto, Leandro Cruz Santana, Karine Souza Santos and Emerson Ticona Fioretto

Department of Morphology, Federal University of Sergipe, Av. Marechal Rondon, s/n - Jd. Rosa Elze, São Cristóvão - SE, Brazil

*Corresponding author: Klank FA, Department of Morphology, Federal University of Sergipe, Av. Marechal Rondon, s/n - Jd. Rosa Elze, Sao Cristovao - SE, Brazil, Tel: 55793194-6625; E-mail: franciscoklank@ymail.com

Received: May 07, 2018; Accepted: June 05, 2018; Published: June 07, 2018

Copyright: ( $) 2018$ Klank FA et al. This is an open-access article distributed under the terms of the creative commons attribution license, which permits unrestricted use, distribution, and reproduction in any medium, provided the original author and source are credited.

\begin{abstract} SHG.

Objective: To evaluate the histopathological changes in human placentas and umbilical cords of patients with

Methods: The sample consisted of 28 pregnant women with SHG, divided into 4 groups: Normal Pregnant Women, Gestational Hypertension, Chronic Hypertension, and the group with pre-eclampsia, totaling 7 pregnant women per group. The data were analyzed by the statistical program Grad pad prism, using the one-way Anova and Toukey's multiple comparison test. The placentas and umbilical cords were submitted to 4 different methodologies, beginning with the histological processing - light microscopy, then histopathological analyzes of placentas by Hematoxylin and Eosin - HE, histopathological analyzes of the placentas following the staining technique with Masson and histopathological analyzes of the placentas. placentas following the Coloring Technique - PAS.
\end{abstract}

Results: The age of pregnant women in the pregnancy cycle is between 24 and 29 years. The weight of normotensive pregnant women was $(72 \mathrm{~kg})$, gestational hypertension $(85 \mathrm{~kg})$, chronic hypertension $(82 \mathrm{~kg})$, preeclampsia $(81 \mathrm{~kg})$. The height of the pregnant women was around $(1.60 \mathrm{~cm})$. The anatomical aspects of the newborns were observed; $(3.44 \mathrm{~kg})$, gestational hypertension $(3.46 \mathrm{~kg})$, chronic hypertension $(3.12 \mathrm{~kg})$, and preeclampsia $(3.14 \mathrm{~kg})$. Regarding height, it was observed that in neonates of normotensive pregnant women, the mean was $(48.35 \mathrm{~cm})$, gestational hypertension $(47.78 \mathrm{~cm})$, chronic hypertension $(42.00 \mathrm{~cm})$, and pre-eclampsia $(34.57 \mathrm{~cm})$. The thoracic perimeter of newborns of normotensive pregnant women is $33.57 \mathrm{~cm}$, with gestational hypertension $(33.00 \mathrm{~cm})$, with chronic hypertension $(31.85 \mathrm{~cm})$ and with preeclampsia $(31.78 \mathrm{~cm})$. The gestational age of normotensive pregnant women was 37 weeks, gestational hypertension, 39 weeks, and chronic hypertension, at 36 weeks. The placenta size of normotensive pregnant women $(63.8 \mathrm{~cm})$, gestational hypertension $(52.4 \mathrm{~cm})$, chronic hypertension $(54.7 \mathrm{~cm})$ and preeclampsia $(57 \mathrm{~cm})$. The weight of placenta of normotensive pregnant women $(0.72 \mathrm{~g})$, gestational hypertension $(0.65 \mathrm{~g})$, chronic hypertension $(0.49 \mathrm{~g})$, and preeclampsia $(0.63$ g).

Conclusion: Pregnant women in adulthood acquired SHG; the newborns of pregnant women with chronic hypertension were those that suffered the greatest impact of the syndrome, presenting slight decrease in weight, height, thoracic perimeter and cephalic perimeter. The placentas and umbilical cords of pregnant women with SHG presented structural changes and involvement of the tissue structures when compared to the normotensive pregnant women group. In this sense, it is concluded that gestational attachments that have important data on gestational development and can contribute actively to the clinical data of pregnant and newborn.

Keywords: Gestational hypertension; Chronic hypertension; Preeclampsia

\section{Introduction}

Gestational hypertensive syndromes are one of the causes responsible for increased morbidity and mortality rates in Latin America, including Brazil; high morbidity and mortality rates due to GSHs have been reported in the North and Northern regions, suggesting a high incidence of GSHs in the North and Northeast regions compared to that in the Southeast, South, and Center-West regions [1].

GHS classification includes gestational hypertension, pre-eclampsia, eclampsia, chronic hypertension $(\mathrm{CH}), \mathrm{CH}$ with superimposed preeclampsia, and HELLP syndrome. GHS may be diagnosed in up to $10 \%$ of pregnancies worldwide [2]. Though several studies have been conducted on GHS in Brazil and worldwide, the disease and its complications continue to occur; thus, new possibilities of treatments and detection are sought to minimize its effects [3].

Scientific evidence, for example, the histopathological studies of the placenta and umbilical cord of pregnant women, indicates that the detection of GHS enables the elucidation of structural abnormalities at the tissue and cellular level and to deduce intrauterine and perennial connections that may reveal new strategies of treatment [4]. Therefore, the first guiding question arises, "Hence, we hypothesized that the SGA can alter the structure and functional capacity of the gestational annexes, directly impacting maternal and neonatal health. 
Page 2 of 5

Although several published studies have associated histopathology to gestational diseases, studies have not been conducted in the Sergipe region (specifically, in the municipality of Aracaju, where high-risk pregnancies are reported. Thus, the histological analysis of the placental changes is necessary visualize the symptoms of GHS in the human placenta of pregnant women in the Northeast region of Brazil. Therefore, the general objective was to evaluate the histopathological changes in the placenta and umbilical cord samples of participants with hypertensive syndromes during pregnancy.

\section{Materials and Methods}

The study was conducted at the Nossa Senhora de Lourdes Maternity, a reference hospital in the State of Sergipe, Brazil. It is a highly complex unit, which serves women with high-risk pregnancies through the Unified Health System. The clientele are pregnant women with hypertension, diabetes, heart disease, or preterm labor [5].

The project was approved by Plataforma Brazil (Opinion: 679.337). All protocols of the research undertaken complied with the recommendations proposed by the National Health Council, through Resolution 466/2012, which presents the guidelines and norms regulating research involving human beings [6].

The participants were recruited when they visited the Our Lady of Lourdes Maternity Hospital. The researchers were allotted different tasks, such as screening and collecting the initial information of the participants and family members Free-willed informed consent was obtained after the research objective was explained, and the researcher collected the information and transcribed it in the socio-demographic questionnaire under the following heads: maternal age, marital status, housing ownership conditions, schooling, occupation and origin; gestational age; obstetric history (gestation, parity, and abortion); and maternal, fetal, and neonatal obstetric complications and type of delivery.

For this research, the data of the pregnant women were recorded, such as age, height, and weight; the data of the neonate were also used, such as height, birth weight, and cephalic, thoracic, and abdominal perimeters.

\section{Diagnosis of the disease}

The diagnosis of gestational hypertension and its clinical derivations were recorded in the patient's chart. In addition, the information contained in the medical record was compiled with data from the International Classification of Diseases-10 and the American Congress of Obstetricians and Gynecologists, which defines GHS from the classification pregnancy-induced hypertension (PIH) or pregnancyspecific hypertension (PSH) is hypertension after 20 weeks' gestation with proteinuria, and pre-eclampsia (PE), induced by $\mathrm{PIH}$, is defined as hypertension with the presence of proteinuria while superimposed pre-eclampsia (SPE) is $\mathrm{CH}$ aggravated during pregnancy due to proteinuria; eclampsia (CE) is a combination of comatose eclampsia and HELLP syndrome (hemolysis, thrombocytopenia, and elevated liver enzymes) [2].

In this study, 28 pregnant women with GHS were divided into the following four groups: gestational hypertension, $\mathrm{CH}$, pre-eclampsia, and control groups, with seven participants per group.

\section{Transport of biological material}

The placental attachments were collected after delivery and transported to the Federal University of Sergipe (UFS), Brazil, to the Morphology Department, to perform the histological analysis and determine the placental weight.

There were 28 placenta samples from pregnant women attended to in the high-risk maternity ward, of whom seven were normotensive participants that constituted the control group.

The placenta samples were collected after the second stage of labor sand were stored in Styrofoam boxes under ice and transported to the Laboratory of Morphology, UFS. Subsequently, by a random principle, fragments from different regions of both the fetal and maternal portions were collected for histological processing, following a standard protocol to obtain histological sections. They were then sent to the Pathology Department of the University Hospital/UFS for subsequent staining with hematoxylin and eosin (HE), Masson trichrome, and periodic acid-Schiff (PAS).

The placenta samples from the participants of the control group and those with GHS were analyzed under the microscope under normal conditions.

\section{Histological processing}

Light microscopy: The specimen for light microscopy was included in paraffin based on standard techniques. The specimen was then serially sectioned into a $5-\mu \mathrm{m}$ microtome and stained with HE, Masson trichrome, and PAS based on standard techniques.

For histological processing, the tissue slices were washed in distilled water and kept in $70 \%$ alcohol for $12 \mathrm{~h}$. During the histological processing, the specimen was dehydrated using an increasing gradient of ethanol (95\%, Absolute I and II), immersed for $30 \mathrm{~min}$ each and diaphanized in two consecutive Xilol baths for $15 \mathrm{~min}$ each. The specimen was then embedded in paraffin (Synth) diluted 1:1 with xylene for $30 \mathrm{~min}$ in the oven at $60^{\circ} \mathrm{C}$, followed with pure paraffin for 1 $\mathrm{h}$ at $60^{\circ} \mathrm{C}$. The specimen was then included in aluminum foils containing pure paraffin and kept at room temperature after being properly identified.

Cuts of $5-\mu \mathrm{m}$ thickness were obtained on a rotating microtome and collected on histological slides coated with balsam. For the morphological analysis, the sections were stained with HE, Masson, and PAS, for later observation under light field microscopy.

Finally, the slides of the control, gestational hypertensive, $\mathrm{CH}$, and pre-eclampsia groups were photo-documented, using the image capture system coupled to the Leica microscope, model DM500 with an ICC50 model camera. The images obtained were analyzed histopathologically [5].

Histopathological analyses of placenta samples by HE: The biological material of the placental tissue of this study was collected and fixed in $10 \%$ formalin and included in paraffin following a standard histological processing technique for subsequent $\mathrm{HE}$ staining.

The placenta and umbilical cord sections used in the microscopic evaluation were randomized, extracting portions of the placental disks from the peripheral, intermediate, and central thirds.

HE staining was used to visualize the structural changes of the lesions and tissue reaction. 
The sections were hydrated and stained with hematoxylin for $8 \mathrm{~min}$, then submerged in tap water for $20 \mathrm{~min}$ and re-submerged in $0.5 \%$ eosin for $5 \mathrm{~min}$. Subsequently, they were dehydrated in sol of increasing alcohol and diaphanized in xylol; the slides were covered with salve and cover slip for later observation under light field microscopy. Subsequently, the histological sections were examined under an optical microscope, always by the same observer [5].

Histopathological analyses of the placental samples following masson trichome staining: The solutions were prepared and processed as follows: Bouin's solution was first prepared with $75 \mathrm{ml}$ saturated solution of picric acid, $25 \mathrm{ml}$ pure formaldehyde, and $5 \mathrm{ml}$ glacial acetic acid.

Subsequently, Weigertiron hematoxylin was prepared with two different solutions: solution A (1 g hematoxylin powder and $100 \mathrm{ml}$ $95 \%$ alcohol) and solution B (4 $\mathrm{ml} 29 \%$ aqueous solution of ferric chloride, $95 \mathrm{ml}$ distilled water, and $1 \mathrm{ml}$ hydrochloric acid concentrate), which were combined in equal parts of both solutions A and $\mathrm{B}$.

Subsequently, the Biebrich Scarlet solution was prepared with $90 \mathrm{ml}$ $1 \%$ aqueous Biebrich Scarlet solution, $10 \mathrm{ml} 1 \%$ aqueous fuchsin acid solution, and $1 \mathrm{ml}$ glacial acetic acid, followed by preparation of phosphotungstic-phosphomolybdic acid solution, with $2.5 \mathrm{~g}$ phosphotungstic acid, $2.5 \mathrm{~g}$ phosphomolybdic solution, and $100 \mathrm{ml}$ distilled water.

In addition, the aniline blue solution was prepared with $2.5 \mathrm{~g}$ aniline blue, $2 \mathrm{ml}$ glacial acetic acid, and $100 \mathrm{ml}$ distilled water, and finally the water-acid solution was prepared with $100 \mathrm{ml}$ distilled water and $1 \mathrm{ml}$ glacial acetic acid.

The samples were dewaxed and hydrated, washed in running water for $5 \mathrm{~min}$, and submerged in Bouin's solution for $1 \mathrm{~h}$ in the oven at $60^{\circ} \mathrm{C}$. Subsequently, the samples were washed in running water until the yellow left by the Bouin's solution disappeared, then they were washed with distilled water, stained with Weigertiron hematoxylin solutions (A and B) for $10 \mathrm{~min}$, rinsed in running water for $10 \mathrm{~min}$, redissolved in distilled water, and stained with Biebrich Scarlet solution for $5 \mathrm{~min}$. Subsequently, the samples were washed with distilled water and differentiated with phosphotungsticphosphomolybdic acid solution for $10 \mathrm{~min}-15 \mathrm{~min}$.

The slides were then washed with distilled water and stained with aniline blue solution for $5 \mathrm{~min}-10 \mathrm{~min}$, then washed again in distilled water and submerged in $1 \%$ glacial acetic acid solution for $3 \mathrm{~min}-5$ min. Finally, the slides were passed again in distilled water and dehydrated, diaphanized, and mounted with balsam and coverslips [5].

Histopathological analysis of the placenta samples following the colouring technique with PAS: The slides were heated in $200 \mathrm{ml}$ distilled water in a $500 \mathrm{ml}$ Erlenmeyer flask to boiling. Subsequently, 1 g basic fuchsin (Merck, granulated) was added carefully and gradually, avoiding thermal reaction, after allowing the temperature to drop $<60^{\circ} \mathrm{C}$, and was filtered at room temperature. Subsequently, $20 \mathrm{~m} \mathrm{l} 1 \mathrm{~N}$ hydrochloric acid, $92 \mathrm{ml}$ distilled water, $8 \mathrm{ml}$ hydrochloric acid, and $1 \mathrm{~g}$ sodium metabisulfite were added. After a 3-h interval, $1 \mathrm{~g}$ activated carbon was added. After PAS dye preparation, the slides were dewaxed, hydrated, submerged, oxidized in $0.5 \%$ periodic acid for $15 \mathrm{~min}$, washed in running water for $5 \mathrm{~min}$, and rinsed in distilled water thrice. Subsequently, the slides were stained in Schiff's reagent for $30 \mathrm{~min}$, rinsed again in running water for $5 \mathrm{~min}$, and stained with Harris hematoxylin for $5 \mathrm{~s}$. They were again washed in running water for 5 min, and finally dehydrated, diaphanized and mounted with coverslips and balsam [5].

\section{Statistical analysis}

Initially, all variables were analyzed descriptively. The quantitative variables were analyzed by observing the minimum and maximum values and calculating the means and standard deviation. Statistical analyses were performed using the GraphPad software (Prism 5.0) using the analysis of variance and Tukey test, with $\mathrm{p}<0.5$ being significantly different.

\section{Results and Discussion}

GHS is defined by a systolic pressure $\geq 140 \mathrm{mmHg}$ and a diastolic pressure $>90 \mathrm{mmHg}$, and characterized by its absence after termination of pregnancy, but it can persist in severe cases, which can then progress into complex cases $[7,8]$.

The age of the pregnant women with GHS in the study is 26 years to 29 years. Data evidenced in the scientific literature indicate that the development of GHS occurs in women who become pregnant in adulthood or in extreme ages [9]. Previous studies are in agreement with the data found and show that the most prevalent age group in women in the gestational period is 20 years- 34 years, considered the most adequate reproductive age [10-12].

Previous research conducted $[13,14]$ showed that the economic and social dynamics of pregnant women have been changing over the years, and a new social context has begun to emerge, allowing them to opt for late pregnancy, close to 40 years of age. This fact described in the scientific literature reveals that the higher the age of pregnant women, the greater the propensity to develop hypertension.

Other determinant factors for the occurrence and development of GHS are those related to the weight of the pregnant woman, showing that weight gain and obesity before gestation and during gestation actively contribute to GHS onset. Eating habits, hypersomnia, and hypercaloric and hypoproteinic diets are associated with weight gain [15].

In this context, [16] data showed that the highest nutritional problem in Brazil is overweight in the gestational period, not malnutrition, with data between $25 \%$ and $30 \%$. In addition, other factors are related to the development of GHS, such as diabetes, hypertension, nephropathies, family or personal history of preeclampsia or eclampsia, low educational level, and work activity outside home, $\mathrm{AB}$ blood group, primigravida, multiple gestations, fetal hydrops, and trophoblastic neoplasia [17].

Regarding the literature, the variable weight of the neonate, determines the normality from $3200 \mathrm{~g}$; Thus, in our results, we found that the weight was smaller only in the groups with $\mathrm{CH}$ and preeclampsia compared with the literature [18]. However, no difference was found between the groups for this variable.

The neonates were born with weight within the standard of normality. Another research points out that the weight of neonates of pregnant women with GHSwas > $2500 \mathrm{~g}$, [19] showing that these data re in agreement with the data evidenced in the research in question.

The scientific literature determines the normality for the variable height from $50.0 \mathrm{~cm}$ [18]. In our study, we observed that the neonates of all groups were smaller compared with the literature, which may be influenced by non-measurable variables, such as mother's nutrition, 
pre-natal care, type of delivery, and genetic and epidemiological values [20]. In our study, we only observed the difference for the chronic hypertensive group compared with the other groups.

Literature shows that the mean cephalic (CP) and thoracic perimeters (TP) are between $33 \mathrm{~cm}$ and $35 \mathrm{~cm}$ and between $31 \mathrm{~cm}$ and $33 \mathrm{~cm}$, respectively [18]. Data from the current study are within the normal range; however, no research has correlated fetal variables to GHS. Therefore, this research contributes to the understanding of how syndromes can influence and/or impact the body of neonates.

Therefore, the research notes that changes in embryonic development caused by maternal diseases, such as GHS, may result in malformations, ranging from small asymmetries and/or anomalies with greater functional and aesthetic impairments. The causes are usually linked to multifactorial diseases that precede birth and can be inherited or acquired [21].

Gestational age was another finding found in the study. The gestational age of participants with normotension, gestational hypertension, $\mathrm{CH}$, and pre-eclampsia who visited the Hospital NossaSenhora de Lourdes was 37,39,36, and 37 weeks, respectively.

All the participants subdivided into the groups were observed to be admitted to the maternity unit at nine months of gestation, although the data presented a significant variance between the groups (due to the weekly differences between each group). Although all participants delivered at nine months of gestation, labor occurred at 36 weeks in the group with $\mathrm{CH}$, suggesting that $\mathrm{CH}$ accelerated labor.

The literature reports that the concept of prematurity includes every neonate delivered $<37$ completed weeks of gestation ( $<259$ days), counted from the first day of the last menstrual period $[22,23]$.

The classification of prematurity can be understood considering gestational age, birth weight, and comparison of weight to gestational age. Based on scientific literature, prematurity can be classified as borderline (neonates between 35 weeks and 36 weeks of gestation, weighing between $2200 \mathrm{~g}$ and $2800 \mathrm{~g}$, with stature of $45 \mathrm{~cm}-46 \mathrm{~cm}$ ), moderate (neonates between 30 weeks and 34 weeks, weighing between $1600 \mathrm{~g}$ and $2600 \mathrm{~g}$, with stature of $39 \mathrm{~cm}-44 \mathrm{~cm}$ ), and extreme (neonates before the 30th week of gestation, with weight $<150 \mathrm{~g}$ ) [18].

Another important point to note is the size and weight of the placenta. However, no study to date has correlated the size and weight of the placenta with GHS, making these data novel.

In addition, the data found for $\mathrm{CP}$ and $\mathrm{TP}$ were not statistically different. No studies have correlated CP and TP to gestational disease. Thus, the present study contributes to this gap.

With regard to the histological analysis of the umbilical cord in the control group, the vein and artery with the intima and muscular layers were observed to gradually thicken. The umbilical cord of neonates of most participants with GHS showed smaller arterial and venous lumens, such as slowing the physiological changes between mother and fetus, which may affect the body structure of the neonate, such as weight and height.

The placenta samples of participants without hypertensive syndromes were found to be mature, deciduous, and flat with eosinophilic tissue and presence of fibrin, with dense epithelium and intact villi. The chorionic villi of the mature placenta of the normal pregnant women were quite vascularized, with knots and scions.
Generally, the histological findings of the placental analysis of normal pregnant women were compatible with the descriptions of the microscopic anatomy of the normal placenta found in the literature. In a review study on the microscopic anatomy of the normal placenta, intense capillarization were also observed in the chorionic villi and arteries present in the decidua that also presented aspect and blood vessels with delicate walls, without thickening. The chorial membranes are also intactin the external area [24].

In this context, thickening of the vessel wall seems to be a histological marker of systemic changes in maternal health with a reflex in placental development. In this study, thickening of the umbilical cord vasculature wall has been commonly observed in all participants with any hypertensive syndromes, reflecting some degree of difficulty in blood flow. Vessel hyalinization, with microcalcification points, was also a common finding, being more abundant, however, in the more severe syndromes Please check that the revision has not altered your intended meaning.

In the placenta samples of patients with gestational hypertension, aged areas and areas of hyaline degeneration in the villi were also observed in addition to the above findings. Maturation is a rapid reflex because an acute pathological response to increased pressure levels was also observed in this study in the placenta samples of pregnant women with gestational hypertension.

They also found that thickening of the muscular layer of the placental vessels is a characteristic feature of the placenta of pregnant women with hypertension and also associated with higher prevalence of hyalinized vessels in placenta of pregnant women with hypertension (44.2\%) compared with normotensive pregnant women and without hypertensive complications during gestation (3.1\%). Although not exclusively found in the placenta of hypertensive pregnant women, a higher number of microscopic infarcts was also observed as a characteristic feature (9.3\% versus $3.2 \%)$ [4]

In the cases of pregnant women with severe eclampsia, our study showed that global severe clusters with foci of calcification, difficulty of visualization of the cells, aged cells, and visualization of severely diminished trophoblastic cells were the most common findings. Histology could not identify the differentiation of trophoblast cells, observed as process of generalized cell aging, present hyalinization, calcification, villus grouping in vessels, as well as totally degenerate decidua and placental hemorrhage.

\section{Conclusion}

In a comparative study of the pathological anatomy of the placenta samples of participants with severe pre-eclampsia and those with severe preeclampsia complicated by the HELLPsyndrome,26deciduous arteriopathy was observed, evidenced by deposition of fibrinoid material with necrosis of the vessel wall and ectasia of the vessel lumen. Accelerated maturation was also observed. This change was found in $31.25 \%$ of placenta samples studied and was not affected by the HELLP syndrome [25].

A high frequency of fibrinoid necrosis and hypertrophy of the tunica media of placental vessels in the placenta of patients with preeclampsia was also found [26]. A high prevalence of the reduction of the space between the villi and villi surface was found in pregnant women with preeclampsia, while a significant fibrin deposit was more frequently observed in the placenta samples of participants with gestational hypertension and $\mathrm{CH}$ [27]. 
In addition to findings similar to those of this study (early maturation, villus hypoplasia, vessel thickening, and decidua arteriopathy), placental perfusion problems correlated these findings, supporting other studies [28].

From the above, the areas of hyaline degeneration in the villi were changes frequently seen in all syndromes studied, prevailing in both mature and immature areas. Similarly, thickening of vessels due to proliferation of the tunica media and fibrin deposition was also a common abnormality. Immature and edematous villi, with few vessels and areas with edema and decreased vessel numbers were frequent in the placenta samples of participants with gestational hypertension, and the intense grouping of degenerated villi was quite prevalent in participants with pre-eclampsia.

The observed changes may reflect some damage on the external surface. Low birth weight and low Apgar scores, due to the lower blood supply as a result of hypertensive syndromes, were frequently correlated in fetuses of participants with hypertension, whose placenta possessed changes similar to those described in the present study [25].

However, although some of the lesions frequently found and described in literature are not specific to hypertensive syndromes, these lesions were observed to increase in number in the placenta samples of participants with hypertension compared to that in the placenta samples of those without hypertensive syndromes during gestation [4].

Our study showed that GHSs were developed in adult pregnant women, with a mean age of 24 years -29 years, with medium height, and showing no signs of gestational obesity.

The neonates of pregnant women with $\mathrm{CH}$ can be possibly identified because of a slight decrease in weight, height, and thoracic and cephalic perimeters compared with other groups with gestational diseases and data in the scientific literature.

The placenta and umbilical cord samples presented changes in the tissue structures compared with to those in the control group, and we can affirm that GHS directly impacted the embryonic annexes, completely modifying standard structures.

\section{References}

1. Souza PJ (2015) Maternal mortality and the new objectives of sustainable development. Rev Bras Ginecol Obstet 37: 549-551.

2. The American Congress of Obstetricians and Gynecologists (2016) Washington: AOCG 2:1.

3. Peraçoli JC, Parpinelli MA (2005) Hypertensive disorders of pregnancy: Identification of cases serious. Rev Bras Ginecol Obstet 27: 627-634.

4. Artico LG, Madi JM, Godoy AEG, Coelho CP, Rombaldi RL, et al. (2009) Histopathological changes in human placentas related to hypertensive syndromes. Rev Bras Ginecol Obstetr 31: 10-16.

5. Campinas State University. (2011) Techniques employed in the department of anatomic pathology. Campinas: Unicamp. 2: 1.

6. National Health Council Resolution (2012) Approves the guidelines and regulations for research on human development. 2: 1 .

7. Assis TR, Viana FP, Rassi S (2008) Risk factors for hypertension in pregnancy. Arq Bras Cardiol 91: 11-17.

8. Dusse LMSA, Vieira LM, Carvalho MG (2001) Review of hemostatic changes in the specific hypertension of pregnancy. J Bras Patol Med 37: 267-272.
9. Moura MDR, Castro MP, Margotto PR, Rugolo LMSS (2011) Arterial hypertension: Importance of follow-up in the neonatal outcome. Communical Health 22: 113-120.

10. Carmo TA, Nitrini SMOO (2004) Prescription of medicines for pregnant women: A pharmacoepidemiological study. Cad Saúde Pública 20: 1004-1013.

11. Salvador BC, Paula HAA, Souza CC, Cota AM, Batista MA, et al. (2008) Prenatal care in vicosa-MG: Contributions to the discussion of public health policies. Rev Med Minas Gerais 18: 167-174.

12. KroeffL R, Mengue S, Schmidt MI, Duncan BB, Favaretto ALF, et al. (2004) Associated factors are not the same as those suggested by Brazilian societies. Rev Saúde Pública 38: 261-267.

13. Ribeiro MP, Ribeiro AB, Stabile Neto C, Anção MS, Saragoça MA, et al. (1982) Prevalence of arterial hypertension in the workforce of greater são paulo: Influence of age, sex and ethnicity. Rev Assoc Med Brazil 28: 209-211.

14. Mulrow CD, Chiquette E, Ferrer RL, BM Sibai, KR Stevens, et al. (2000) Management of chronic hypertension during pregnancy. Rockville: AHRQ. 2: 1.

15. Vítolo MR, Good MSF, Range CM (2011) Impact of a dietary guidelines program on the weight gain rate of pregnant women attended to in health units. Rev Bras Ginecol Obstet 33: 14-19.

16. Stulbach TE, Benício MHD, Andreazza R, Kono S (2007) Determining the gain weight excessive during pregnancy in public service of prechristmas of low risk. Rev Bras Epidemiol 10: 99-108.

17. Piirainen T, Isolauri E, Lagström H, Laitinen K (2006) Impact of dietary counselling on nutrient intake during pregnancy: A prospective cohort study. Br J Nutr 96: 1095-1104.

18. Calil VMLT, Leone CR, Tronchin DMR (1996) Characterization of the newborn infant. In: Leone CR. Assistance integrated with the newborn. São Paulo: Atheneu pp: 79-95.

19. Chaim SRP, Oliveira SMJV, Kimura AF (2008) Pregnancy: Induced hypertension and the neonatal outcome. Acta Paul Enferm 21: 53-58.

20. Santos RS, Dias IMV (2005) Reflecting on congenital malformation. Rev Bras Enferm 58: 592-596.

21. Bramham K, Parnell B, Nelson-Piercy, Seed PT, Poston L, et al. (2014) Chronic hypertension and pregnancy outcomes: systematic review and meta-analysis. BMJ 348: 2301.

22. Olsen P, Laara E, Rantakallio P, Jarvelin MR, Sarpola A, et al. (1995) Epidemiology of preterm delivery in two birth cohorts with an interval of 20 years. Am J Epidemiol 142: 1184-1193.

23. Hall MH, Danielian P, Lamont RF (1997) The importance of preterm birth. In: Elder MG, Lamont RF, Romero R (eds). Preterm labor, New York, USA. Churchill Livingstone pp: 1-28.

24. Huppertz B (2008) The anatomy of the normal placenta J Clin Pathol 61: 1296-1302.

25. Mehrabian F, Mohammadizadeh F, Moghtaderi N, Najafian A (2012) Comparison of placental pathology between severe preeclampsia and HELLP syndrome. Arch Gynecol Obstet 285: 175-181.

26. Stevens DU, Al-Nasiry S, Bulten J, Spaanderman ME (2013) Decidual vasculopathy in preeclampsia: lesion characteristics relate to disease severity and perinatal outcome. Placenta 34: 805-809.

27. Corrêa RR, Gilio DB, Cavellani CL, Paschoini MC, Oliveira FA, et al. (2008) Placental morphometrical and histopathology changes in the different clinical presentations of hypertensive syndromes in pregnancy. Arch Gynecol Obstet 277: 201-206.

28. Veerbeek JH, Nikkels PG, Torrance HL, Gravesteijn J, Post Uiterweer ED, et al. (2014) Placental pathology in early intrauterine growth restriction associated with maternal hypertension. Placenta 35: 696-701. 